QUARTERLY OF APPLIED MATHEMATICS

VOLUME LXIII, NUMBER 3

SEPTEMBER 2005, PAGES 455-467

S $0033-569 X(05) 00969-X$

Article electronically published on August 17, 2005

\title{
FUNDAMENTAL AND SINGULAR SOLUTIONS OF LAMÉ EQUATIONS FOR MEDIA WITH ARBITRARY ELASTIC ANISOTROPY
}

\author{
BY
}

SERGEY V. KUZNETSOV

Institute for Problems in Mechanics, Moscow 119526, Russia

\begin{abstract}
Fundamental and singular solutions of Lamé equations for media with arbitrary elastic anisotropy are constructed on the basis of multipolar expansions (expansions in spherical harmonics) of symbols and the corresponding operators. Theorems of convergence are formulated. A posteriori error estimates are presented.
\end{abstract}

1. Introduction. Solution of the boundary-value problems by boundary integral equation methods needs fundamental solutions. For isotropic elastic medium, solution of equations of equilibrium in the 3-dimensional case was constructed by Thomson (Lord Kelvin) [51. Presumably, the first attempt for justification of Kelvin's heuristic procedure for the fundamental solution construction was undertaken by Somigliana [45. Much later, the main properties of the fundamental solution were studied by a specially developed limiting process by Sternberg et al. [48, [49] and Turteltaub and Sternberg [54.

The first attempt to construct fundamental solutions for anisotropic media goes back to Fredholm [14] and Zeilon [58, who used the Fourier transformation for symbol construction, but failed in the inverting of the Fourier transformation. By use of the Fourier transformation and Levi's method for reducing the number of parameters, the fundamental solutions in a closed form were obtained by Kupradze and Basheleishvili [25], Burchuladze [7, 8], Rizzo and Shippey [42, and Hatiashvili [15] for the two-parametric problems, when the three-dimensional displacement field depends upon two spatial variables (for media with monoclinic symmetry these are plane and anti-plane problems). Construction of parametrics for cylindrically anisotropic axially symmetric bodies was done by Kuznetsov [28.

Analytical solutions for some specific kinds of elastic anisotropy in the 3-dimensional case were constructed by Kröner [24, Basheleishvili [1, and Kahniashvili 21]. In the

Received June, 2004.

2000 Mathematics Subject Classification. Primary 35E05; 74S15.

Key words and phrases. Fundamental solution, singular solution, Lamé equations, multipolar series, spherical harmonics.

(C)2005 Brown University 
case of weak anisotropy, asymptotic formulas for fundamental solution were obtained by Leibfrid [34. Some properties of the fundamental solution for media with arbitrary elastic anisotropy, but without its explicit construction, were studied by Sáenz [43. It should be noted that analytical expressions for the fundamental solution in $\mathbb{R}^{3}$ were obtained only for one narrow subclass of elastic orthotropy, which includes transverse isotropy.

Two principally different numerical methods are used for constructing fundamental solutions in the 3-dimensional case and media with arbitrary elastic anisotropy: (i) the method based on Radon's transformation, and (ii) the multipolar decomposition method.

Methods based on Radon's transformation were proposed by Lifshits and Rozentsveig [35]. The analogous method was later used by Synge [50, who exploited McConnell's approach [38. See also Willis [56]; Vogel and Rizzo [55]; Wilson and Cruse [57]; and Natroshvili [40], whose work along with constructing fundamental solution, contains theoretical analysis of normal solvability and the Fredholm properties of the boundary integral operators. Radon's transformations were also used by Kapanadze 22], Deb, Henry and Wilson [11, and Schclar [4], whose work along with Radon's transformations contains description of a new method for solving boundary integral equations based on the theorem of duality for singular solutions. Sometimes the method based on Radon's transformations is referred to as the plane wave decomposition method; see John [20]. Numerical experiments carried out by Wilson and Cruse [57] showed that this method is highly time-consuming from a computational point of view. To decrease the computing time, these authors proposed to use spline approximations on the unit sphere.

Analysis of theoretical problems for potential type operators related to the second order elliptic differential equations and the corresponding boundary integral operators goes as far back as Somigliana [45], Fredholm [14, and Tricomi [53. For matrix differential operators of anisotropic elasticity, the corresponding analysis was developed by Hatiashvili [15]; see also Burchuladze and Gegelia [9]; Kuznetsov [29]; Natroshvili [40], Jentsch and Natroshvili [17; Jentsch, Natroshvili and Wendland [18, 19.

Construction of the fundamental solution can also be done by the multipolar expansions (expansions in spherical harmonics on the unit sphere). The idea of such expansions is due to Fredholm 14 and Zeilon 58. However, lack of the appropriate technique on inversion of the Fourier transforms of (homogeneous) symbols corresponding to operators with weak singularity did not allow researchers to obtain final expressions for the fundamental solutions at that time. Similar difficulties were observed by Kroner 24, Kinoshita and Mura 23] and Bross [4, 5], 6]. Some ideas for the use of harmonic polynomials to aid in the construction of the fundamental solution were given by Bezier [2].

The complete theory of multipolar expansions for construction of singular operators was developed by Calderon and Zygmund [10] and Mikhlin [39]. Analogous theory for operators with weak singularity was developed by Bochner [3]. Later, methods of multipolar decompositions were extrapolated by Kuznetsov [27, 30] for construction of the fundamental solutions for equations of equilibrium, equations of free vibrations (Kuznetsov, [32]) and construction of some singular solutions of mechanics which correspond to kernels 
of the double-layer potentials and their derivatives (Kuznetsov, 30, 31]). Numerical algorithms based on the multipolar expansions are discussed by Perelmuter and Kuznetsov [41. Theoretical considerations show (see Kuznetsov, 27, [30]) that the multipolar expansion method has an advantage in computation time as compared with methods based on Radon's transformation.

The following analysis is concerned with theoretical and numerical aspects of the multipolar expansion method for constructing fundamental and singular solutions of equations of equilibrium for 3D media with arbitrary elastic anisotropy.

2. Basic notations. For an anisotropic elastic medium, equilibrium equations in terms of the displacement field can be written in the form:

$$
\mathbf{A}\left(\partial_{x}\right) \mathbf{u}(\mathbf{x}) \equiv-\operatorname{div}_{x} \mathbf{C} \cdot \nabla_{x} \mathbf{u}(\mathbf{x})=0,
$$

where $\mathbf{A}$ is the $3 \times 3$ matrix differential operator of the equilibrium equations; $\mathbf{u}$ is the displacement vector field, and $\mathbf{C}$ is the fourth-order elasticity tensor, assumed to be positive definite:

$$
\underset{\mathbf{A} \in \mathbb{R}^{3} \otimes \mathbb{R}^{3}, \mathbf{A} \neq 0}{\forall \mathbf{A}} \mathbf{A} \mathbf{C} \cdot \mathbf{A}>0 .
$$

The medium itself is assumed to be homogeneous and hyperelastic. The latter ensures symmetry of the elasticity tensor regarded as an operator acting in the six-dimensional space of symmetric second-order tensors:

$$
\underset{\mathbf{A}, \mathbf{B} \in \operatorname{sym}\left(\mathbb{R}^{3} \times \mathbb{R}^{3}\right)}{\forall \mathbf{A}} \mathbf{A} \cdots \mathbf{C} \cdot \mathbf{B}=\mathbf{B} \cdots \mathbf{C} \cdot \mathbf{A} .
$$

Conditions (2) and (3) allow us to decompose the elasticity tensor (regarded as the linear operator in the 6 -dimensional space of symmetric second-order tensors in $\mathbb{R}^{3}$ ) in the following tensorial product:

$$
\mathbf{C}=\sum_{\alpha=1}^{6} \lambda_{\alpha} \mathbf{S}_{\alpha} \otimes \mathbf{S}_{\alpha}, \quad \lambda_{\alpha}>0,
$$

where $\mathbf{S}_{\alpha}$ are symmetric second-order tensors forming an orthonormal set with respect to Euclidian norm:

$$
\mathbf{S}_{\alpha} \cdot \mathbf{S}_{\beta}=\delta_{\alpha \beta} .
$$

In (5), $\delta_{\alpha \beta}$ is the Kronecker symbol.

Application of the integral Fourier transform

$$
g^{\wedge}(\xi)=\int_{\mathbb{R}^{3}} g(\mathbf{x}) \exp (-2 \pi i \mathbf{x} \cdot \xi) d x, \quad \xi \in \mathbb{R}^{3}
$$

to the differential operator in Eq. (11) gives the matrix symbol of the operator A:

$$
\mathbf{A}^{\wedge}(\xi)=(2 \pi)^{2} \xi \cdot \mathbf{C} \cdot \xi .
$$

It follows from (2) and (3) that symbol $\mathbf{A}^{\wedge}$ is strongly elliptic:

$$
\underset{\mathbf{a}, \xi \in \mathbb{R}^{3}, \mathbf{a}, \xi \neq 0}{\forall \mathbf{a}, \xi} \mathbf{a} \cdot \mathbf{A}^{\wedge}(\xi) \cdot \mathbf{a}>0 .
$$

Expression (6) also shows that symbol $\mathbf{A}^{\wedge}$ is (positive) homogeneous of degree 2 and analytic in $\mathbb{R}^{3}$. 
The definition of the fundamental solution implies the following identity:

$$
\mathbf{A}^{\wedge}(\xi) \cdot \mathbf{E}^{\wedge}(\xi)=\mathbf{I},
$$

where $\mathbf{E}^{\wedge}$ is the symbol of the fundamental solution, and $\mathbf{I}$ is the unit (diagonal) matrix. The identity (8) allows us to represent the symbol $\mathbf{E}^{\wedge}$ in the form

$$
\mathbf{E}^{\wedge}(\xi)=\mathbf{A}_{0}^{\wedge}(\xi) / \operatorname{det} \mathbf{A}^{\wedge}(\xi),
$$

where $\mathbf{A}^{\wedge}{ }_{0}(\xi)$ is the cofactor of the symbol $\mathbf{A}^{\wedge}(\xi)$. Expression (9) shows that symbol $\mathbf{E}^{\wedge}$ is also strongly elliptic, positively homogeneous of degree -2 , and $\mathbf{E}^{\wedge}(\xi)=C^{\infty}\left(\mathbb{R}^{3} \backslash 0 ; \mathbb{R}^{3} \otimes\right.$ $\left.\mathbb{R}^{3}\right)$.

Fourier transform inversion applied to expression (9) gives

Proposition 2.1. The fundamental solution $\mathbf{E}$ of the equilibrium equations (11) is positively homogeneous of degree -1 and $\mathbf{E}(\mathbf{x}) \in C^{\infty}\left(\mathbb{R}^{3} \backslash 0 ; \mathbb{R}^{3} \otimes \mathbb{R}^{3}\right)$.

3. Multipolar expansions for fundamental solution. Let symbol $\mathbf{E}^{\wedge}$ be expanded in a series of spherical harmonics on the unit sphere $S \subset \mathbb{R}^{3}$ :

$$
\mathbf{E}^{\wedge}(\xi)=|\xi|^{-2} \sum_{n=0,2, \ldots}^{\infty} \sum_{k=-n}^{n} \mathbf{E}_{n k} Y_{n}^{k}\left(\xi^{\prime}\right), \quad \xi^{\prime}=\xi /|\xi|,
$$

where $Y_{n}^{k}$ are spherical harmonics of degree $n$ and of the order $k$, connected to the associated Legendre functions $P_{n}^{k}$ in the following way:

$$
Y_{n}^{k}\left(\xi^{\prime}\right)=\left(\frac{(2 n+1)}{4 \pi} \frac{(n-|k|) !}{(n+|k|) !}\right)^{1 / 2} \times P_{n}^{|k|}(\cos \theta) \times e^{i k \varphi} .
$$

In expression (11), spherical and Cartesian coordinates are connected by the following relation:

$$
\xi^{\prime}=(\sin \theta \sin \varphi ; \sin \theta \cos \varphi ; \cos \theta) .
$$

Formulas for computing the associated Legendre functions are presented in the Appendix. It should be noted that spherical harmonics (11) form the complete orthonormal set in $L^{2}(S)$. Matrix coefficients $\mathbf{E}_{n k}$ in (10) are determined by integration over the unit sphere in $\mathbb{R}^{3}$ :

$$
\mathbf{E}_{n k}=\int_{S} \mathbf{E}^{\wedge}\left(\xi^{\prime}\right) Y_{n}^{k}\left(\xi^{\prime}\right) d \xi^{\prime},
$$

where $d \xi^{\prime}=\sin \theta d \theta d \varphi$ is the Lebesgue measure induced on $S$.

REMARK 3.1. Summation in (10) is done on harmonics of the even order only. That is because: (i) the symbol $\mathbf{E}^{\wedge}$ is strongly elliptic, and (ii) expressions (8) and (9) ensure positive homogeneity of the symbol. Indeed, spherical harmonics of the even order are even on $S$, while harmonics of the odd order are odd: $Y_{n}^{k}\left(-\xi^{\prime}\right)=-Y_{n}^{k}\left(\xi^{\prime}\right)$.

Proposition 3.1 (Kuznetsov, 27). The series in (10) converges to the symbol $\mathbf{E}^{\wedge}$ : a) in a weak topology in $\mathfrak{T}\left(\mathbb{R}^{3} ; \mathbb{R}^{3} \otimes \mathbb{R}^{3}\right)$, where $\mathfrak{T}^{\prime}$ is the space of tempered distributions in $\left.\mathbb{R}^{3} ; \mathrm{b}\right)$ in $\left.L^{2}\left(S ; \mathbb{R}^{3} \otimes \mathbb{R}^{3}\right) ; \mathrm{c}\right)$ in $C_{c}\left(\mathbb{R}^{3} \backslash 0 ; \mathbb{R}^{3} \otimes \mathbb{R}^{3}\right)$, where $C_{c}$ stands for topology of the compact convergence in $\mathbb{R}^{3} \backslash 0$. 


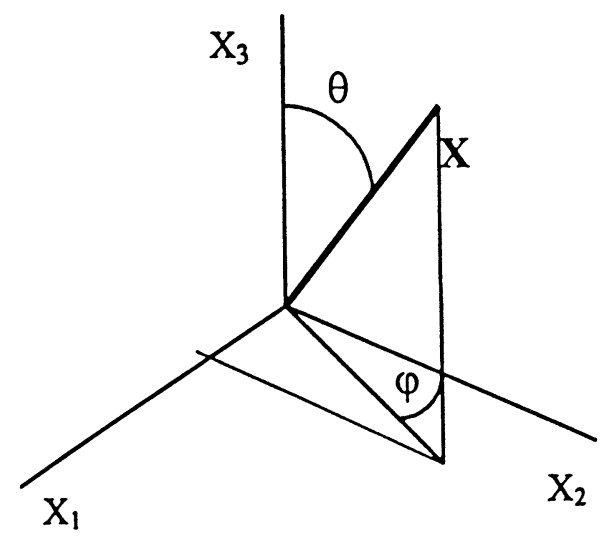

FIG. 1.

Proof of Proposition 3.1 follows directly from the right-hand side of (9), ensuring symbol $\mathbf{E}^{\wedge}$ to be positive homogeneous of degree -2 and (real) analytic everywhere in $\mathbb{R}^{3} \backslash 0$.

Now, application of Bochner's inverting formula (see Stein, Ch. III, §3, Theorem 5) to terms in (10) gives the fundamental solution also obtained in the form of multipolar series:

$$
\mathbf{E}(\mathbf{x})=|\mathbf{x}|^{-1} \sum_{n=0,2, \ldots}^{\infty} \gamma_{n} \sum_{k=-n}^{n} \mathbf{E}_{n k} Y_{n}^{k}\left(\mathbf{x}^{\prime}\right), \quad \mathbf{x}^{\prime}=\mathbf{x} /|\mathbf{x}|,
$$

where parameters $\gamma_{n}$, known as Bochner's multipliers, can be easily computed by the following formula (Stein, Ch. III, §3):

$$
\gamma_{n}=(-1)^{n / 2} \pi^{1 / 2} \frac{\Gamma\left(\frac{n+1}{2}\right)}{\Gamma\left(\frac{n+2}{2}\right)} .
$$

Expression (13) and Stirling's expansion for the $\Gamma$-function allow us to obtain the following asymptotic formula:

$$
\left|\gamma_{n}\right|=O(1), \quad n \rightarrow \infty .
$$

Since the Fourier transformation sets topological isomorphism in the spaces $\mathfrak{T}^{\prime}\left(\mathbb{R}^{3} ; \mathbb{R}^{3} \otimes\right.$ $\left.\mathbb{R}^{3}\right), L^{2}\left(S ; \mathbb{R}^{3} \otimes \mathbb{R}^{3}\right)$ and it is continuous in the space $C_{c}\left(\mathbb{R}^{3} \backslash 0 ; \mathbb{R}^{3} \otimes \mathbb{R}^{3}\right)$, Proposition 3.1 implies:

Proposition 3.2. The series on the right side of (12) converges to the fundamental solution: a) in the weak topology in $\mathfrak{T}^{\prime}\left(\mathbb{R}^{3} ; \mathbb{R}^{3} \otimes \mathbb{R}^{3}\right) ;$ b) in $L^{2}\left(S ; \mathbb{R}^{3} \otimes \mathbb{R}^{3}\right)$; c) in $C_{c}\left(\mathbb{R}^{3} \backslash 0 ; \mathbb{R}^{3} \otimes \mathbb{R}^{3}\right)$.

REMARK 3.2. a) The following notation for correspondence between Cartesian coordinates of the affine vector $\mathbf{x}$ and its spherical coordinates $\theta, \varphi$ will be needed: $\theta=$ $\arccos \left(\frac{x_{3}}{|\mathbf{x}|}\right), \varphi=\arcsin \left(\frac{x_{1}}{\left|\mathbf{x}^{\prime}\right|}\right)$, where $\mathbf{x} \equiv\left(x_{1} ; x_{2} ; x_{3}\right)$ and $\mathbf{x}^{\prime}=\left(x_{1} ; x_{2}\right)$; refer to Fig. 1. These formulas for angles $\theta, \varphi$ are valid for any $\mathbf{x} \neq 0$; 
b) It is known that summation of a harmonic series with coefficients having small errors may lead to an ill-posed problem in the specific pairs of metric spaces. To overcome possible difficulties, a regularization method was proposed by Kuznetsov [27. However, numerical experiments with some real anisotropic materials (see Perelmuter and Kuznetsov, 41]) revealed relatively fast convergence of multipolar series and numerical stability, so there was no need in applying regularization techniques.

4. Multipolar expansions for singular operators. When (i) the stress field produced by a single-layer potential, or (ii) the displacement field produced by the doublelayer potential are needed, appropriate spatial derivatives can be obtained directly from the multipolar expansions of the principle symbol corresponding to the composition of the surface traction operator and the fundamental solution (when such a composition is restricted to the surface where the density is located, this produces a singular operator).

Application of the Fourier transformation to the composition

$$
\mathbf{V}(\mathbf{y}) \equiv \mathbf{T}\left(\nu_{y}, \partial_{y}\right) \cdot \mathbf{E}(\mathbf{y}),
$$

where $\mathbf{T}\left(\nu_{y}, \partial_{y}\right) \equiv \nu_{y} \cdot \mathbf{C} \cdot \nabla_{y}$ is the surface traction operator, produces the following matrix principle symbol:

$$
\mathbf{V}^{\wedge}(\mathbf{y}, \xi)=2 \pi i\left(\nu_{y} \cdot \mathbf{C} \cdot \xi\right) \cdot \mathbf{E}^{\wedge}(\xi) .
$$

In (15), (16), $\nu_{y}$ denotes the field of the unit normals to the supporting surface (the surface where the potential densities are located). In obtaining (16), the usual technique of "freezing" coefficients is used.

REMARK 4.1. a) Kernel (15) produces important types of operators, called the doublelayer potentials (see Kupradze et al., Ch. II, §4):

$$
\mathbf{V}[\mathbf{f}](\mathbf{x}) \equiv \int_{\partial \Omega} \mathbf{f}(\mathbf{y}) \cdot\left(\mathbf{T}\left(\nu_{y}, \partial_{y}\right) \cdot \mathbf{E}(\mathbf{x}-\mathbf{y})\right) d y,
$$

where $\partial \Omega$ is a 2 -dimensional piecewise smooth manifold in $\mathbb{R}^{3} ; \mathbf{f}$ is an integrable on the $\partial \Omega$ function, and $d y$ is the Lebesgue measure concentrated on $\partial \Omega$. It should be noted that field $\nu_{y^{\prime}}$ is defined only for regular points of $\partial \Omega$. When $x \in \partial \Omega$, the integral in (17) is evaluated as the Principle Value (P.V.) integral. The right-hand side of (17) contains the conormal derivative along $\nu_{y}$, while in applications it is often desirable to exclude it. Applying a regularization technique to the principle symbol of the operator $\mathbf{V}$ yields the desired symbol (see Kuznetsov, [33]):

$$
\mathbf{V}^{\prime \wedge}\left(\mathbf{y}, \xi^{\prime}\right)=\mathrm{P} . \mathrm{V} \cdot \int_{-\infty}^{\infty} \nu_{y} \cdot \mathbf{C} \cdot \xi \otimes \mathbf{E}^{\wedge}(\xi) d \xi^{\prime \prime}, \quad \mathbf{y} \in \partial \Omega,
$$

where $\xi=\xi^{\prime}+\xi^{\prime \prime} \nu_{y} ; \xi^{\prime} \in \prod_{\nu_{y}}\left(\prod_{\nu_{y}}\right.$ is the plane tangential to $\partial \Omega$, and $\left.\xi^{\prime \prime}=\xi \cdot \nu_{y}\right)$.

b) In the subsequent analysis, kernels and the corresponding operators will be denoted by the same letter.

Some obvious properties of the principle symbol $\mathbf{V}^{\wedge}$ are stated in the following proposition: 
Proposition 4.1. At any fixed regular point $\mathbf{y} \in \partial \Omega$, the principle symbol $\mathbf{V}^{\wedge}(\mathbf{y}, \xi)$ is a) real-analytic in $\mathbb{R}^{3} \backslash 0$ with respect to the $\xi$-variable; (b) homogeneous of degree -1 with respect to the $\xi$-variable, but not positive homogeneous.

Multipolar expansion of this symbol yields

$$
\mathbf{V}^{\wedge}(\mathbf{y}, \xi)=|\xi|^{-1} \sum_{n=1,3, \ldots}^{\infty} \sum_{k=-n}^{n} \mathbf{V}_{n k}(\mathbf{y}) Y_{n}^{k}\left(\xi^{\prime}\right), \quad \xi=\xi /|\xi|
$$

where matrix coefficients $\mathbf{V}_{n k}$ are determined by integration of the symbol $\mathbf{V}^{\wedge}$ on the unit sphere:

$$
\mathbf{V}_{n k}(\mathbf{y})=\int_{S} \mathbf{V}^{\wedge}\left(\mathbf{y}, \xi^{\prime}\right) Y_{n}^{k}\left(\xi^{\prime}\right) d \xi^{\prime}
$$

The series on the right-hand side of (18) contains harmonics of the odd order only; this follows from Proposition 4.1,b and Remark 3.1.

Proposition 4.2. a) At any fixed regular point $\mathbf{y} \in \partial \Omega$, the series on the right-hand side of (18) converges to the symbol $\mathbf{V}^{\wedge}$ : a) in the weak topology in $\left.\mathfrak{T}^{\prime}\left(\mathbb{R}^{3} ; \mathbb{R}^{3} \otimes \mathbb{R}^{3}\right) ; \mathrm{b}\right)$ in $L^{2}\left(S ; \mathbb{R}^{3} \otimes \mathbb{R}^{3}\right) ;$ c) in $C_{c}\left(\mathbb{R}^{3} \backslash 0 ; \mathbb{R}^{3} \otimes \mathbb{R}^{3}\right)$.

Proof of Proposition 4.2 is analogous to the proof of Proposition 3.1

Application of Bochner's inverting formula to the terms in expansion (18) gives kernel $\mathrm{V}$ :

$$
\mathbf{V}(\mathbf{y}, \mathbf{x})=|\mathbf{x}|^{-2} \sum_{n=1,3, \ldots}^{\infty} \gamma_{n}^{\prime} \sum_{k=-n}^{n} \mathbf{V}_{n k}(\mathbf{y}) Y_{n}^{k}\left(\mathbf{x}^{\prime}\right), \quad \mathbf{x}^{\prime}=\mathbf{x} /|\mathbf{x}|,
$$

where coefficients $\mathbf{V}_{n k}$ depend upon "frozen" variable $\mathbf{y}$, and multipliers $\gamma_{n}^{\prime}$ admit the following representation [46, Ch. III, §3]:

$$
\gamma_{n}^{\prime}=i^{n} \pi^{-1 / 2} \frac{\Gamma\left(\frac{n+2}{2}\right)}{\Gamma\left(\frac{n+1}{2}\right)} .
$$

Comparing (18), (19) shows that the left-hand side of (19) is real-valued and real-analytic in $\mathbb{R}^{3} \backslash 0$.

Similar to the fundamental solution, the multipolar series (19) has the following properties:

Proposition 4.3. At any fixed regular point $\mathbf{y} \in \partial \Omega$, the series on the right-hand side of (19) converges to the distribution defined by its kernel $\mathbf{V}$ : a) in the weak topology in $\left.\mathfrak{T}^{\prime}\left(\mathbb{R}^{3} ; \mathbb{R}^{3} \otimes \mathbb{R}^{3}\right) ; \mathrm{b}\right)$ in $\left.L^{2}\left(S ; \mathbb{R}^{3} \otimes \mathbb{R}^{3}\right) ; \mathrm{c}\right)$ in $C_{c}\left(\mathbb{R}^{3} \backslash 0 ; \mathbb{R}^{3} \otimes \mathbb{R}^{3}\right)$.

REMARK 4.2. a) The distribution identified with its kernel $\mathbf{V}$ and acting on functions with the non-thin support in $\mathbb{R}^{3}$ represents an operator with weak (integrable) singularity, while restriction of this operator on functions having support on a two-dimensional manifold forces us to regard it as a singular operator;

b) From a computational point of view, it is more convenient to expand in multipolar series the tensorial symbol

$$
\mathbf{V}^{\prime \prime \wedge}(\xi)=2 \pi i(\mathbf{C} \cdot \xi) \cdot \mathbf{E}^{\wedge}(\xi)
$$


which is the three-valent symbol. Expanding such a symbol into multipolar series, then applying Bochner's inverting formula, and making convolution with the field of the unit normals $\nu$, produces the desired kernel $\mathbf{V}$.

5. Multipolar expansions for hyper-singular operators. In this section, construction of the composition of the surface traction operator and the operator of the double-layer potential is regarded:

$$
\mathbf{W}(\mathbf{z}, \mathbf{y}, \mathbf{x}) \equiv \mathbf{T}\left(\nu_{x}, \partial_{x}\right) \cdot \mathbf{V}^{t}(\mathbf{y}, \mathbf{x}),
$$

where $\mathbf{W}$ is the kernel of the corresponding pseudodifferential (hyper-singular) operator; the upper index $t$ stands for transposition of the matrix-valued operator $\mathbf{V}$.

"Freezing" variables and applying Fourier transformation to (22) produces the following amplitude (see [52, Ch. 1, $\S 2$ for the definition of amplitudes):

$$
\mathbf{W}^{\wedge}(\mathbf{z}, \mathbf{y} ; \xi)=-(2 \pi)^{2}\left(\nu_{z} \cdot \mathbf{C} \cdot \xi\right) \cdot \mathbf{E}^{\wedge}(\xi) \cdot\left(\xi \cdot \mathbf{C} \cdot \nu_{y}\right) .
$$

In obtaining (23), we assigned different variables appearing in the "frozen" coefficients in (16) and (22).

Routine procedure, similar to one exploited for obtaining (17), (19), produces the kernel $\mathbf{W}$ in the form of multipolar series:

$$
\mathbf{W}(\mathbf{z}, \mathbf{y}, \mathbf{x})=|\mathbf{x}|^{-3} \sum_{n=0,2, \ldots}^{\infty} \gamma_{n}^{\prime \prime} \sum_{k=-n}^{n} \mathbf{W}_{n k}(\mathbf{z}, \mathbf{y}) Y_{n}^{k}\left(\mathbf{x}^{\prime}\right), \quad \mathbf{x}^{\prime}=\mathbf{x} /|\mathbf{x}|,
$$

where in contrast to (17), (19), the series on the right-hand side of (24) contains harmonics of the even order only, because of positive homogeneity of the amplitude with respect to the $\xi$-variable. Matrix coefficients $\mathbf{W}_{n k}$ are determined by integration of the amplitude (23) on the unit sphere in $\mathbb{R}^{3}$ similarly to obtaining $\mathbf{V}_{n k}$.

Bochner's multipliers $\gamma_{n}^{\prime \prime}$ in (24) have the following form:

$$
\gamma_{n}^{\prime \prime}=(-1)^{n / 2} \pi^{-3 / 2} \frac{\Gamma\left(\frac{n+3}{2}\right)}{\Gamma\left(\frac{n}{2}\right)} .
$$

The following Proposition is analogous to Proposition 4.3

Proposition 5.1. At any fixed regular points $\mathbf{y}, \mathbf{z} \in \partial \Omega$, the series on the right-hand side of (24) converges to the distribution associated with its kernel $\mathbf{W}$ : a) in the weak topology in $\mathfrak{T}^{\prime}\left(\mathbb{R}^{3} ; \mathbb{R}^{3} \otimes \mathbb{R}^{3}\right) ;$ b) in $L^{2}\left(S ; \mathbb{R}^{3} \otimes \mathbb{R}^{3}\right) ;$ c) in $C_{c}\left(\mathbb{R}^{3} \backslash 0 ; \mathbb{R}^{3} \otimes \mathbb{R}^{3}\right)$.

REMARK 5.1. a) At fixed regular points $\mathbf{z}, \mathbf{y} \in \partial \Omega$, operator $\mathbf{W}$ acting on functions with the non-thin support in $\mathbb{R}^{3}$ becomes an operator with strong (non-integrable) singularity. The kernel of the operator $\mathbf{W}$ can be represented in the form

$$
\mathbf{W}(\mathbf{z}, \mathbf{y}, \mathbf{x})=\mathbf{W}_{0}(\mathbf{z}, \mathbf{y}) \delta(\mathbf{x})+\mathbf{W}_{1}(\mathbf{z}, \mathbf{y}, \mathbf{x}), \quad \mathbf{z}, \mathbf{y} \in \partial \Omega, \quad \mathbf{x} \in \mathbb{R}^{3},
$$

where $\mathbf{W}_{0}$ is a matrix piecewise-smooth function defined on the manifold $\partial \Omega ; \delta(\mathbf{x})$ is the delta-function in $\mathbb{R}^{3}$; and $\mathbf{W}_{1}$ is the kernel of a singular operator (with respect to the $\mathrm{x}$-variable) having zero mean value:

$$
\underset{\mathbf{z}, \mathbf{y} \in \partial \Omega}{\forall \mathbf{y} \in \mathbf{y}} \int_{S} \mathbf{W}_{1}\left(\mathbf{z}, \mathbf{y}, \mathbf{x}^{\prime}\right) d x^{\prime}=0
$$


Condition (27) is due to decomposition (26) where the first term on the right-hand side is responsible for non-zero mean value.

b) Restriction of the operator $\mathbf{W}$ on functions having support on the manifold $\partial \Omega$ forces us to regard it as the hyper-singular operator. By analogy with Remark 4.1,a, we can define

$$
\mathbf{W}[\mathbf{f}](\mathbf{x})=\text { F.P. } \int_{\partial \Omega} \mathbf{T}\left(\nu_{x}, \partial_{x}\right) \cdot\left(\mathbf{f}(\mathbf{y}) \cdot\left(\mathbf{T}\left(\nu_{y}, \partial_{y}\right) \mathbf{E}(\mathbf{x}-\mathbf{y})\right)\right) d y, \quad \mathbf{x} \in \partial \Omega,
$$

where the corresponding hyper-singular integral is evaluated as the finite part (F.P.) integral (see [12, Ch. 5, §5). An example of the explicit regularization technique for evaluating the F.P. integral is given in (Kuznetsov, 31], 33]).

c) From a computational point of view, it is more convenient to expand the following tensorial symbol in the multipolar series:

$$
\mathbf{W}^{\prime \wedge}(\xi)=-(2 \pi)^{2} \mathbf{C} \cdot\left(\xi \otimes \mathbf{E}^{\wedge}(\xi) \otimes \xi\right) \cdot \mathbf{C},
$$

where $\mathbf{W}^{\prime \wedge}$ is the four-valent symbol. Applying Bochner's inverting formula to thus obtained multipolar series and then taking convolution from both sides with the vector fields $\nu_{z}, \nu_{y}$ produces the desired kernel $\mathbf{W}$.

6. Error estimates. Suppose that: (i) multipolar series (10) and (12) are truncated and contain harmonics of degree up to $N$; and, (ii) matrix coefficients $\mathbf{E}_{n k}$ contain computational errors, except coefficients $\mathbf{E}_{00}$ (solid harmonic), which is assumed to be defined precisely. Substituting such a truncated expansion for the fundamental solution in the equations of equilibrium (11) yields

$$
\mathbf{A}\left(\partial_{x}\right) \mathbf{E}_{N}(\mathbf{x})=\delta(\mathbf{x}) \mathbf{I}+\mathbf{K}_{N}(\mathbf{x}),
$$

where $\mathbf{E}_{N}(\mathbf{x})$ is the approximate fundamental solution due to truncation and computational errors, and $\mathbf{K}_{N}(\mathbf{x})$ is a matrix residual operator.

REMARK 6.1. The approximate fundamental solution $\mathbf{E}_{N}(\mathbf{x})$ is of class -2 in $\mathbb{R}^{3}$; this follows from the multipolar expansions (10), (12). Now, the left-hand side of (29) ensures that operator $\mathbf{K}_{N}$ is of class 0 in $\mathbb{R}^{3}$.

Proposition 6.1. Operator $\mathbf{K}_{N}$ on the right-hand side of (29) is a singular integral operator in $\mathbb{R}^{3}$.

Proof. Fourier transformation applied to both sides of (29) gives

$$
\mathbf{K}_{N}^{\wedge}(\xi)=\mathbf{A}^{\wedge}(\xi) \cdot\left(\mathbf{E}^{\wedge}(\xi)-\mathbf{E}_{N} \wedge(\xi)\right) .
$$

In obtaining (30), relation (8) was used. Since the right-hand side of (30) is positive homogeneous of degree 0 , and the operator $\mathbf{K}_{N}$ satisfies condition of zero mean value on the unit sphere (due to (29) and the condition (ii) preceding (29)), $\mathbf{K}_{N}$ appears to be the singular operator in $\mathbb{R}^{3}$.

The Plancherel theorem applied to the operator $\mathbf{K}_{N}$ shows that its (operator) $L^{2}$ norm can be majored by the corresponding $L^{\infty}$-norm of the symbol $\hat{\mathbf{K}_{N}}$ (see, 46, Ch. II, $\S 1$, n. 1.4). Both norms are equivalent for scalar operators. Due to homogeneity of 
degree 0 of the symbol $\hat{\mathbf{K}_{N}}$, it is natural to evaluate the $L^{\infty}$-norm of this symbol on the unit sphere $S \subset \mathbb{R}^{3}$.

Let $K_{N}^{p q}$ denote the $p, q$-component of the symbol $\hat{\mathbf{K}_{N}}$ :

$$
\begin{gathered}
K_{N}^{p q}\left(\xi^{\prime}\right)=\delta^{p q}-(2 \pi)^{2} \sum_{\substack{n=0,2, \ldots \\
p=-n}}^{N} \sum_{k=1,2,3,}^{p r s t} \frac{\xi_{r} \xi_{s}}{|\xi|^{2}} \widetilde{E}_{n k}^{t q} Y_{n}^{k}\left(\xi^{\prime}\right) \\
p, q=1,20
\end{gathered}
$$

where $\widetilde{E}_{n k}^{t q}$ are corresponding coefficients of the fundamental solution $\mathbf{E}_{N}$. Introducing scalar

$$
\Delta_{N}=\sup _{1 \leq p \leq 3} \sup _{\xi^{\prime} \in S}\left(\sum_{q=1}^{3}\left|K_{N}^{p q}\left(\xi^{\prime}\right)\right|\right),
$$

which majorizes the spectral $L^{\infty}$-norm of the symbol $\hat{K_{N}}$ (see, [37], Ch. III, $§ 1.6 .5$ ), we obtain a natural estimate for precision of the numerically-constructed fundamental solution $\mathbf{E}_{N}$. In fact, due to (29), (32) and Plancherel's theorem, we have $\Delta_{N} \geq\left\|\mathbf{K}_{N}\right\|_{\left(L^{2}, L^{2}\right)}=$ $\left\|A\left(\partial_{x}\right) \mathbf{E}_{N}-\delta \mathbf{I}\right\|_{\left(L^{2}, L^{2}\right)}$, where $\left(L^{2}, L^{2}\right)$ is the corresponding operator norm.

Numerical analysis developed by Kuznetsov [27] showed that multipolar series rather rapidly converge for the tested kinds of elastic anistropy. For example, a good accuracy with the error estimate $\Delta_{N} \approx 10^{-5}$ has been achieved for the following orthotropic material:

$$
\begin{array}{r}
C^{1111}=1.091 ; C^{1122}=2.429 ; C^{1133}=5.036 ; \\
C^{2222}=1.209 ; C^{2233}=1.210 ; \\
C^{3333}=1.091 ; \\
C^{1212}=0.082 ; C^{2323}=0.068 ; C^{3131}=0.074
\end{array}
$$

by the truncated series $(N=6)$ corresponding to 49 spherical harmonics. Computations were carried out with the real numbers having mantissa with $\sim 15$ decimal digits. Higher accuracy with $\Delta_{N} \approx 10^{-8}$ was achieved at $N=12$, while further increasing parameter $N$ up to 20 harmonics did not reveal any improvements of the accuracy, because of the irreducible computational errors.

Acknowledgements. The author thanks Professor Gregory Rodin for valuable information, the Russian Fund for Fundamental Research (Grant 04-01-00781) and the Russian Academy of Sciences (Grant for Scientific Program No. 13) for financial support.

\section{Appendix.}

Formulas for Legendre polynomials and functions. The following formula, which is due to Hobson [16], gives Legendre polynomials in the convenient-for-computational-purposes form:

$$
P_{n}(x)=2^{-n} \sum_{k=0}^{E(n / 2)} \frac{(-1)^{k}(2 n-2 k) !}{k !(n-k) !(n-2 k) !} x^{n-2 k}
$$

where $E(n / 2)$ denotes the entire part of $n / 2$. 
On obtaining coefficients of the polynomial $P_{n}(x)$, the associated Legendre functions $P_{n}^{k}$ can be computed by the following analytical formulas:

$$
P_{n}^{k}(x)=(-1)^{k}\left(1-x^{2}\right)^{k / 2} \frac{d^{k}}{d x^{k}} P_{n}(x) .
$$

Combination of (A1) and (A2) produces

$$
\begin{aligned}
P_{n}^{k}(x)=2^{-n}(-1)^{k}\left(1-x^{2}\right)^{k / 2} & \\
& \times \sum_{m=0}^{E(n / 2)-k} \frac{(-1)^{m}(2 n-2 m) !(n-2 m) !}{m !(n-m) !(n-2 m) !(n-2 m-k) !} x^{n-2 m-k} .
\end{aligned}
$$

The associated Legendre functions $P_{n}^{k}$ can also be obtained by combination of two recurrence relations, which are mainly due to Magnus and Oberhettinger [36]:

$$
\begin{gathered}
P_{n+1}^{k}(x)=(n-k+1)^{-1}\left\lfloor(2 n+1) x P_{n}^{k}(x)-(n+k) P_{n-1}^{k}(x)\right\rfloor \\
1 \leq n, \quad 0 \leq k \leq n-1
\end{gathered}
$$

and

$$
\begin{gathered}
\left(1-x^{2}\right)^{1 / 2} P_{n}^{k+2}(x)=2(k+1) x P_{n}^{k+1}(x)-(n(n+1)-k(k+1))\left(1-x^{2}\right)^{1 / 2} P_{n}^{k}(x) \\
0 \leq k \leq n-2
\end{gathered}
$$

where (A4) can be applied for computing $P_{n}^{1}$, while (A5) can be applied for computing $P_{n}^{k}, k>1$.

It can easily be deduced from (A2) that $P_{n}^{n}=1 ; P_{n}^{0}$ coincides with the corresponding Legendre polynomial, and $P_{n}^{k} \equiv 0$ at $k>n$.

\section{REFERENCES}

[1] M. O. Basheleishvili, On fundamental solutions of the differential equations of an anisotropic elastic body (in Russian), Soobsch, Akad. Nauk Gruz. SSR 19, 393-400 (1957). MR0095625 (20:2127)

[2] P. Bézier, Sur quelques propriétés des solutions de problèmes de l'élastostatique linéaire, C. R. Acad. Sci. Paris. A265, 365-367 (1967). MR0224328 (36:7372)

[3] S. Bochner, Harmonic Analysis and the Theory of Probability, Univ. of California Press, Berkeley and Los Angeles, 1955. MR0072370 (17:273d)

[4] H. Bross, Zur Richtungsabhangigkeit physikalischer Eigenschaften in Kristallen mit besonderer Berucksichtung der galvano-und-thermomagnetishen Effekte, Z. Naturforschnung 15a, 859-874 (1960).

[5] H. Bross, Theorie der galvanomagnetischen Erscheinungen bei beliebigen Energiefachen und anisotroper Streuung der Leitungselektronen, Phys. Kondens. Materie 3, 349-373 (1965).

[6] H. Bross, Das Fundamentalintegral der Elastizitätstheorie für kubische Median, ZAMP 19, 434-446 (1968).

[7] T. V. Burchuladze, On some plane boundary-value problems for anisotropic bodies (in Russian), Trudy Tbil. Mat. Inst. 27, 293-332 (1960).

[8] T. V. Burchuladze, Some boundary-value problems for a class of elliptic systems (in Russian), Soobsch. Akad. Nauk Gruz. SSR 31, 513-520 (1963). MR0165217 (29:2506)

[9] T. V. Burchuladze and T. G. Gegelia, Development of the Potential Method in the Elasticity Theory (in Russian), Metsniereba, Tbilisi, 1985. MR0830151 (87f:73013)

[10] A. P. Calderón and A. Zygmund, Singular integral operators and differential equations, Amer. J. Math. 79, 901-921 (1957). MR0100768 (20:7196)

[11] A. Deb, D. P. Henry, Jr., and R. B. Wilson, Alternate BEM formulation for 2- and $3 D$ anisotropic thermoelasticity, Int. J. Solids Struct. 27, 1721-1738 (1991). 
[12] R. E. Edwards, Functional Analysis, Holt, Rinehart and Winston, New York, 1965. MR0221256 $(36: 4308)$

[13] G. Fichera, Existence Theorems in Elasticity. In: Handbuch der Physik, Bd. Via/2, Springer-Verlag, Berlin-Heidelberg-New York, 1972.

[14] I. Fredholm, Sur les équations de l'équilibre d'un corps solide élastique, Acta. Math. 23, 1-42 (1900).

[15] G. M. Hatiashvili, Fundamental solutions of equations of equilibrium for two-dimensional stressstate of anisotropic medium (in Russian), Soobsch. AN Gruz. SSR, 108, 509-512 (1982).

[16] E. W. Hobson, The Theory of Spherical and Ellipsoidal Harmonics, Cambridge University Press, Cambridge, 1931.

[17] L. Jentsch and D. Natroshvili, Non-classical interface problems for piecewise homogeneous anisotropic elastic bodies, Math. Meth. Appl. Sci. 18, 27-49 (1995). MR1313136 (96j:73021)

[18] L. Jentsch, D. Natroshvili, and W. Wendland, General transmission problems in the theory of elastic oscillations of anisotropic bodies (Basic interface problems), I., J. Math. Anal. Appl. 220, 397-433 (1998). MR.1614963 (99d:73043)

[19] L. Jentsch, D. Natroshvili, and W. Wendland, General transmission problems in the theory of elastic oscillations of anisotropic bodies(Mixed interface problems). II., J. Math. Anal. Appl. 235, 418-434 (1999). MR.1703704 (2000f:74029)

[20] F. John, Plane waves and spherical means: Applied to partial differential equations, Springer N.Y., 1955.

[21] N. S. Kahniashvili, On a case of elementary representation of fundamental solutions for differential equations of anisotropic elastic body (in Russian), Trudy Tbil. Univer. 64, 123-126 (1957).

[22] R. V. Kapanadze, Analysis of boundary value problems for anisotropic bodies by potential methods. I. (in Russian), Soobsch. AN Gruz. SSR 87, 82-113 (1987).

[23] N. Kinoshita and T. Mura, On boundary value problem of elasticity, Res. Rep. Fac. Eng. Meiji. Univ. 8, 56-82 (1956).

[24] E. Kröner, Das Fundamentalintegral der anisotropen elastischen Differentialgleichungen, Z. Physik, 136, 402-410 (1953). MR0064606 (16:308e)

[25] V. D. Kupradze and M. O. Basheleishvili, New integral equations of the theory of elasticity of anisotropic bodies (in Russian), Soobsch. Akad. Nauk Gruz. SSR 15, 327-334 (1954). MR0070402 $(16: 1176 \mathrm{~b})$

[26] V. D. Kupradze (editor), T. G. Gegelia, M. O. Basheleishvili, and T. V. Burchuladze, Threedimensional Problems of Mathematical Theory of Elasticity and Thermo-Elasticity (in Russian), Nauka, Moscow, 1976.

[27] S. V. Kuznetsov, Fundamental solutions for Lame's equations in the theory of elasticity (in Russian), Izv. An SSSR. Mech. Tverdogo Tela 4, 50-54 (1989).

[28] S. V. Kuznetsov, Fundamental solutions for equations of equilibrium for cylindrically anisotropic axially symmetric bodies (in Russian), Izv. An SSSR. Mech. Tverdogo Tela 2, 98-102 (1990).

[29] S. V. Kuznetsov, Construction of the Green and Neumann tensor in the theory of elasticity of an anisotropic elastic body (in Russian), Prikl. Mekh. 27 (7), 58-62 (1991). MR1153223 (93c:73014)

[30] S. V. Kuznetsov, Direct boundary integral equation method in the theory of elasticity, Quart. Appl. Math. 53, 1-8 (1995a). MR1315444(95m:73017)

[31] S. V. Kuznetsov, Energy and singular solutions in anisotropic elasticity, C. R. Acad. Sci. Paris, 321 (Ser. IIb), 309-314 (1995b).

[32] S. V. Kuznetsov, Fundamental solutions for equations of harmonic vibrations in the theory of elasticity, C. R. Acad. Sci. Paris 322 (Ser. IIb), 871-878 (1996a).

[33] S. V. Kuznetsov, On the operator of the theory of cracks, C. R. Acad. Sci. Paris 323 (Ser. IIb), 427-432 (1996b).

[34] G. Leibfrid, Versetzungen in anisotropen Material, Z. Physik 135, 23-43 (1953). MR0056451 $(15: 77 \mathrm{~d})$

[35] I. M. Lifshits and L. N. Rozentsweig, On computation of Green tensor for the main equation of elasticity at the case of infinite elastic-anisotropical medium (in Russian), J. Exper. Theor. Phys. 17, 783-791 (1947).

[36] W. Magnus and F. Oberhettinger, Formeln und Sätze für die speziellen Funktionen der mathematischen Physik, Springer-Verlag, Berlin-Göttingen-Heidelberg, 1948. MR0025629 (10:38a)

[37] M. Marcus and H. Mink, A Survey of Matrix Theory and Matrix Inequalities, Allyn and Bacon, Boston, 1964. MR0162808(29:112) 
[38] A. J. McConnell, The hypercircle method of approximation for a system of partial differential equations of the second order, Proc. Roy. Irish Acad. 54A, 263-290 (1951). MR0064272 (16:256b)

[39] S. G. Mikhlin, Singular Integrals and Integral Equations (in Russian), Fizmatgiz, Moscow, 1962.

[40] D. Natroshvili, Mixed interface problems for anisotropic elastic bodies, Georgian Math. J. 2 (6), 631-652 (1995). MR.1357999 (97g:73018)

[41] M. N. Perelmuter and S. V. Kuznetsov, BEM applications to $3 D$ stress analysis of anisotropic composite structures, In: Second Int. Conf. on Composites Engng., New Orleans, 585-586 (1995).

[42] F. J. Rizzio and D. J. Shippey, A method for stress determination in plane anisotropic elastic bodies, J. Comp. Mater. 4, 36-61 (1970).

[43] A. W. Sáenz, Uniformly moving dislocations in anisotropic media, J. Ration. Mech. Anal. 2, 83-98 (1953). MR0052291 (14:601d)

[44] N. A. Schclar, Anisotropic Analysis using Boundary Elements. Topics in Engineering, vol. 20, Computational Mechanics Publications, Southampton 1994.

[45] C. Somigliana, Sopra l'equilibrio di un corpo elastico isotropo, Nuovo Cimento 18, 161-166 (1885)

[46] E. M. Stein, Singular Integrals and Differentiability Properties of Functions, Princeton Univ. Press, Princeton, 1970. MR0290095 (44:7280)

[47] E. M. Stein and G. Weiss, Introduction to Fourier Analysis on Euclidean Spaces, Princeton Univ. Press, Princeton, 1971. MR0304972 (46:4102)

[48] E. Sternberg and R. A. Eubanks, On the concept of concentrated loads and an extension of the uniqueness theorem in the linear theory of elasticity, J. Ration. Mech. Anal. 4, 135-168 (1955). MR0068994 (16:973h)

[49] E. Sternberg and S. Al-Khozaie, On Green's functions and Saint-Venant's principle in the linear theory of viscoelasticity, Arch. Ration. Mech. Anal. 15, 112-146 (1964). MR0158604 (28:1827)

[50] J. L. Synge, The Hypercircle in Mathematical Physics, Cambridge Univ. Press, Cambridge, 1957. MR0097605 (20:4073)

[51] W. Thomson (Lord Kelvin), On the equations of equilibrium of an elastic solid, Cambr. Dubl. Math. J. 3, 87-89 (1848).

[52] F. Treves, Introduction to Pseudodifferential and Fourier Integral Operators, vol. I. Plenum Press, New York and London 1982.

[53] F. Tricomi, Equazioni integrali contenenti il valor principale di un integrale doppio, Math. Z. 27 (1), 87-133 (1927).

[54] M. J. Turteltaub and E. Sternberg, On concentrated loads and Green's functions in elastostatics, Arch. Ration. Mech. Anal. 29, 193-240 (1968). MR0226904 (37:2490)

[55] S. M. Vogel and F. J. Rizzo, An integral equation formulation of three dimensional anisotropic elastostatic boundary value problems, J. Elast. 3, 203-216 (1973).

[56] J. R. Willis, The elastic interaction energy of dislocation loops in anisotropic media, Quart. J. Mech. Appl. Math. 18, 419-433 (1965). MR0193828 (33:2042)

[57] R. B. Wilson and T. A. Cruse, Efficient implementation of anisotropic three dimensional boundaryintegral equation stress analysis, Int. J. Numer. Methods Engng. 12, 1383-1397 (1978). MR0495510 $(58: 14184)$

[58] N. Zeilon, Das Fundamenlintegral der allgemeinen partiellen linearen Differentialgleichung mit Konstanten Koeffizienten, Ark. Matem., Astron. Fys. 6(38), 1-32 (1911). 